\title{
Camada-limite em superfícies lisas e inclinadas: revisitando o método da similaridade
}

\section{Boundary layer on smooth and sloped surfaces: revisiting the similarity method}

\author{
1 Marcus Vinícius Ferreira Soares engemec.marcusvinicius@gmail.com \\ 2 Élcio Nogueira \\ 3 Luiz Cláudio Gomes Pimentel

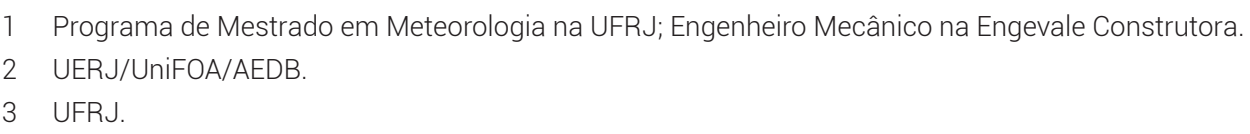

\section{Resumo}

A análise tem como principal objetivo revisar e discutir os princípios do método da similaridade aplicado para camada-limite em superfícies lisas e inclinadas, em regime laminar e regime turbulento. A ênfase se aplica aos aspectos teóricos relacionados com o conceito de similaridade, mas resultados foram obtidos com o objetivo de se comparar com fórmulas empíricas e resultados experimentais. Os aspectos relacionados com regime laminar têm como base o profundo estudo efetuado por Evans (1968) e, em regime turbulento, o texto de Kays e Crawford (1983). Obteve-se resultados para perfis de velocidade e temperatura e grandezas associadas, tais como coeficiente de atrito e número de Stanton, em função do parâmetro de gradiente de pressão e número de Prandtl. Soluções integrais, aproximadas, para camada-limite turbulenta foram implementadas através da equação integral da quantidade de movimento, na qual todas as propriedades do fluido foram consideradas constantes. Objetivo secundário é apresentar os principais aspectos do problema analisado por Falkner e Skan (1931), com foco no método da similaridade. Aplicou-se o método de Runge-Kutta, a partir da expansão em série de potência como primeira aproximação para a solução. Focou-se a apresentação nos procedimentos associados ao método da similaridade, apesar de apresentarem-se resultados, numéricos e gráficos, em número suficiente para enfatizar a sua consistência, quando aplicados para determinação de parâmetros relacionados às camadas-limite térmica e hidrodinâmica, em superfícies lisas.

\section{Palavras-Chave:}

Camada-limite. Superfícies inclinadas. Método da similaridade. Método integral. Camada-limite hidrodinâmica. Camada-limite térmica.

\begin{abstract}
The main objective of the analysis is to revise and discuss the principles of the similarity method applied to the boundary layer on smooth and sloping surfaces, in laminar regime and turbulent regime. The emphasis applies to the theoretical aspects related to the concept of similarity, but results were obtained with the objective of comparing empirical formulas and experimental results. The aspects related to laminar regimen are based on the deep study carried out by Evans (1968), and in turbulent conditions the text of Kays and Crawford (1983). Results were obtained for velocity and temperature profiles, and associated magnitudes, such as friction coefficient and Stanton number, depending on the pressure gradient parameter and Prandt number. Algebraic solutions for turbulent boundary layer were implemented through the integral equation of the amount of movement, where all the properties of the fluid were considered constant. Secondary objective is to present the main aspects of the problem analyzed by Falkner and Skan (1931), focusing on the method of similarity. We apply the Runge-Kutta method, from the series expansion of power as the first approximation to the solution. We focus our presentation more on the procedures associated with the method of similarity, although we present results, numeric and graphs, in sufficient number to emphasize the consistency of them when applied for determining parameters related to the thermal and hydrodynamic boundary layers, on smooth surfaces.
\end{abstract}

\section{Keywords:}

Boundary layer. Sloped surfaces. Similarity method. Integral method. Hydrodynamic boundary layer. Thermal boundary layer.

\section{Como você deve citar?}

SOARES, Marcus Vinícius Ferreira; NOGUEIRA, Élcio; PIMENTEL, Luiz Cláudio Gomes. Camada-limite em superfícies lisas e inclinadas: revisitando o método da similaridade. Cadernos UniFOA, Volta Redonda, n. 41, p. 17-36, dezembro 2019. 


\section{INTRODUÇÃO}

No início do século XX ocorreram importantes desenvolvimentos na teoria hidrodinâmica dos escoamentos sobre corpos, que forneceram explicações para as discrepâncias observadas entre a hidráulica e as equações de Euler para um fluido ideal. As equações de Navier-Stokes já estavam disponíveis, mas o grau de dificuldade imposto, mesmo para problemas simples, e fluidos com baixa viscosidade como o ar e a água, não possibilitavam uma solução satisfatória para as necessidades do momento. Em 1904, Prandtl desenvolveu o conceito de camada-limite, dispondo que o escoamento ao redor de um corpo se divide em duas regiões: uma fina camada próxima ao corpo, onde as tensões viscosas são preponderantes, e uma região externa, onde as forças viscosas podem ser negligenciadas. Através desse "artifício", equações mais simples, derivadas das equações de Navier-Stokes, puderam ser deduzidas e resolvidas com relativa facilidade, para escoamento em placa plana Blasius (1908) e superfícies inclinadas Falkner-Skan (1931), em regime laminar.

A teoria da camada-limite foi aplicada inicialmente para escoamentos laminares e incompressíveis, e os principais trabalhos foram desenvolvidos por Blasius (1908) e Falkner e Scan (1931). 0 método matemático escolhido para solução dessa classe de problemas é denominado "Método da Similaridade", que possibilita transformar uma equação diferencial parcial de $3^{\mathrm{a}}$ ordem não linear, em uma equação diferencial ordinária. Extensões da teoria foram aplicadas para regime turbulento e o conceito de camada-limite, associado ao método da similaridade, continuam sendo utilizados como base para problemas mais complexos do que aqueles analisados por Blasius e Falkner e Scan. Estes últimos encontram-se publicados de forma extensiva nos livros-textos tradicionais. Entretanto, em função da complexidade dos escoamentos turbulentos, uma teoria completa, com base no princípio da similaridade, ainda não existe, e pesquisas continuam sendo realizadas nessa área.

Métodos analíticos de solução de equações diferenciais não lineares parciais, baseados em função de fluxo e método de similaridade, estão sendo aplicados para resolver numerosos problemas de engenharia.

Fluxo bidirecional, incompressível e bidimensional de um fluido micropolar dentro de uma cavidade porosa inclinada na presença de campo magnético é investigado por Nazeer et al. (2017). As equações diferenciais parciais não lineares são resolvidas, empregando-se um esquema robusto de elementos finitos, pelo método de Galerkin.

O problema do escoamento do ponto de estagnação tridimensional estável, incompressível, de um fluido micropolar sobre um disco giratório infinito descentralizado em um meio poroso é estudado Khan et al. (2017). As equações diferenciais parciais são convertidas no conjunto de equações diferenciais ordinárias, utilizando-se a transformação adequada. 0 sistema de equações é analiticamente resolvido por meio de uma técnica não perturbativa, denominado método de análise por homologia (HAM).

Ali et al. (2019) realizaram um estudo numérico para o fluxo de convecção mista dentro de uma cavidade triangular. 0 comportamento reológico do fluido dentro da cavidade é modelado através da equação constitutiva da bi-viscosidade. As equações diferenciais parciais não lineares governantes são discretizadas, usando-se o método dos elementos finitos de Galerkin e a pressão é eliminada através do método de penalidade. Os cálculos são apresentados graficamente para uma ampla faixa do parâmetro de bi-viscosidade, parâmetro de radiação térmica, número de Hartman, número de Grashof, número de Reynolds, parâmetro de geração / absorção de calor e número de Prandtl. 


\section{OBJETIVOS}

A análise tem como principal objetivo revisar e discutir os princípios do método da similaridade aplicado para camada-limite em superfícies lisas e inclinadas, em regime laminar e regime turbulento. A ênfase se aplica aos aspectos teóricos relacionados com o conceito de similaridade, mas resultados foram obtidos com o objetivo de se comparar com fórmulas empíricas e resultados experimentais. Os aspectos relacionados com regime laminar têm como base o profundo estudo efetuado por Evans (1968), e em regime turbulento o texto de Kays e Crawford (1966). Foram obtidos resultados para perfis de velocidade e temperatura e grandezas associadas, tais como coeficiente de atrito e número de Stanton, em função do parâmetro de gradiente de pressão e número de Prandtl.

Assume-se, a princípio, que não há transferência de massa pela superfície e que a componente da velocidade perpendicular a ela é zero (não há transpiração). Além disso, a componente da velocidade paralela à superfície é também nula, condição denominada "sem deslizamento na parede" pela literatura especializada. Todas as soluções e resultados apresentados supõem propriedades constantes, não afetadas pela variação de temperatura, e as velocidades são suficientemente baixas para que o termo de dissipação viscosa possa ser negligenciado.

Com objetivo de comparação, resolveu-se, de forma aproximada, o problema de escoamento turbulento em camada-limite, utilizando-se perfil de velocidade um sétimo (1/7), como uma extensão da solução com gradiente de pressão nulo, apresentada por Kays e Crawford (1983) e Nogueira e Soares (2018). Nesse caso, regime turbulento, a solução integral foi implementada, considerando-se superfícies lisas e as equações deduzidas foram sistematicamente comparadas com resultados empíricos e experimentais.

\section{METODOLOGIA DE SOLUÇÃO}

As equações básicas para as condições de similaridade em camada-limite estão amplamente discutidas por Schlichting (1968), Evans (1968), Kays and Crawford (1983), e Silva Freire (1990), e só os detalhes essenciais para a compreensão dos argumentos são apresentados neste trabalho. Detalhes em Placa Plana encontram-se discutidos por Nogueira e Soares (2018).

Há, na literatura, muitas formas de especificar a existência de soluções similares para as equações de camada-limite. A principal característica associada ao conceito de similaridade em camada-limite é que a distribuição de velocidade não perturbada, do escoamento potencial, deve satisfazer a seguinte expressão, que segue a sugestão original de Falkner-Scan:

$$
U(x)=C x^{m}
$$

$C$ é o valor de $\mathrm{U}(\mathrm{x})$ onde $\mathrm{x}$ é unitário e o valor de $\mathrm{m}$ depende do gradiente de pressão na direção principal do escoamento. Entretanto, de acordo com Spalding (1962), é conveniente impor que U(x) satisfaça a equação:

$$
\frac{d U}{d x}=C U^{\frac{2(\beta-1)}{\beta}}
$$

onde $\beta$ é um parâmetro que mede o gradiente de pressão na direção do escoamento principal. 
A teoria potencial, aplicada ao redor de uma cunha de ângulo $\beta \pi / 2$ (Figura 01), satisfaz a equação 01, acima, onde:

$$
m=\frac{\beta}{(2-\beta)}
$$

e

$$
\frac{1}{\beta} \frac{d U}{d x}=\frac{U}{x} \frac{1}{(2-\beta)}
$$

Entretanto, uma vez que

$$
\frac{d p}{d x}=-\rho U \frac{d U}{d x}
$$

A especificação de $U(x)$ equivale a especificar o gradiente de pressão, que é uma função de $\beta$ :

$$
\frac{d p}{d x}=-\rho U \frac{\beta}{(2-\beta)} \frac{U}{x}
$$

O parâmetro $\beta$, como se pode constatar, depende somente da distribuição de velocidade não perturbada, da variável $x$ ao longo da superfície e do gradiente de pressão.

Interessam as condições de escoamento onde $-0.2 \leq \beta \leq 1.0$, que representam respectivamente, a condição de separação $\beta=-0.2$, e escoamento de estagnação bidimensional $\beta=1.0$ (Figuras 01 e 02).

Figura 1 - Escoamento acelerado para $0<\beta \leq 1.0$

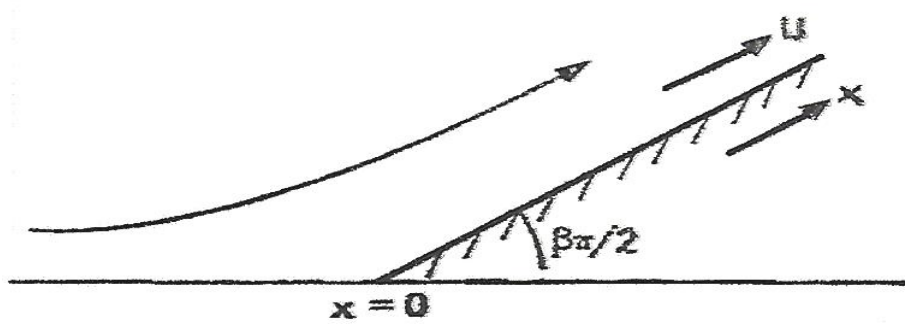

Fonte: autores, 2019 
Figura 2 - Escoamento desacelerado - Beta<1.0.

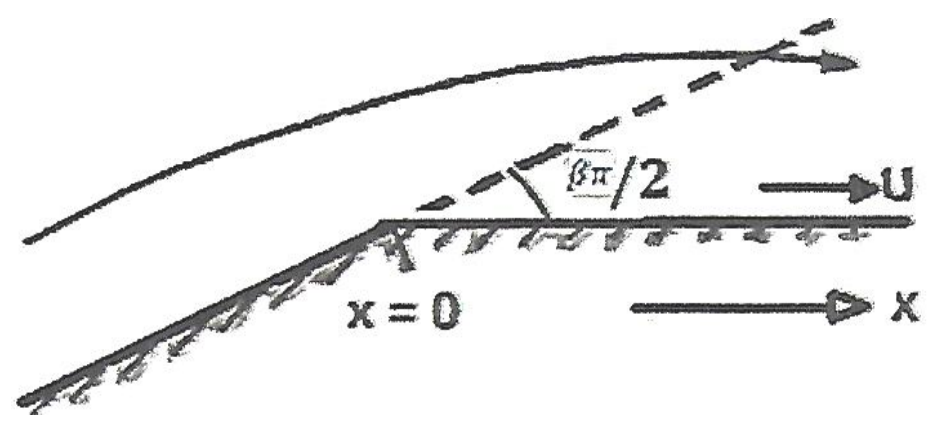

Fonte: autores, 2019

A solução correspondente a $\beta=0$ foi obtida por Blasius, em 1908, através do método de série de potência, e analisada por Nogueira e Soares (2018). A solução estendida, para $\beta \neq 0$, foi obtida por Falkner e Skan (1931). Mangler (1948) generaliza o conceito de similaridade para incluir uma faixa ampliada, em relação aos trabalhos mencionados, para o gradiente de pressão. 0 trabalho de Evans (1968), que envolve um estudo profundo dos trabalhos de Blasius, Falkner e Skan e Mangler, para regime laminar, é uma referência básica para os resultados apresentados à frente. Em regime turbulento, as referências básicas estão relacionadas com as pesquisas realizadas e descritas no texto de Kays e Crawford (1983).

A equação diferencial que governa a distribuição de velocidade em camada-limite similar, dentro da faixa do parâmetro de gradiente de pressão já estabelecido, é dada por por Schlichting (1968), Evans (1968), Kays and Crawford (1983), e Silva Freire (1990):

$$
f^{\prime \prime \prime}+f \cdot f^{\prime \prime}+\beta \cdot\left(1-f^{\prime 2}\right)=0
$$

com as seguintes condições de contorno:

$$
\begin{array}{ll}
\eta=0, & f=f^{\prime}=0 \\
\eta \rightarrow \infty, & f^{\prime} \rightarrow 1.0
\end{array}
$$

$f e \eta$ são definidos por:

$$
\eta=\frac{y}{x} \frac{\left(\frac{U x}{v}\right)^{1 / 2}}{\sqrt{2-\beta}} \text { e } f=\frac{\psi / v}{\left(\frac{U x}{v}\right) \sqrt{2-\beta}}
$$

onde x e y são, respectivamente, as coordenadas primitivas ao longo da superfície e perpendicular a ela.

Ao escolher a coordenada $\eta$ como função de $y / x$, que é muito pequeno, exceto em $\mathrm{x}=0$, pela raiz quadrada do número de Reynolds, $R e_{x}=\left(\frac{U x}{v}\right)^{1 / 2}$, que é muito grande, impomos que $y / x$ é pequeno, mas $\eta$ não é. 
A partir das definições de $\eta$ e $f$, temos expressões para as componentes de velocidades adimensionais:

$$
u=U \frac{d f}{d \eta} \quad e \quad v=-\left(\frac{v}{\beta} \frac{d U}{d x}\right)^{\frac{1}{2}}\left[f+(\beta-1) \eta \frac{d f}{d \eta}\right.
$$

A última condição de contorno, Equação 7.2, significa que, à medida que $\eta$ cresce $f^{\prime}=u / U$, deve se aproximar da unidade, sem excedê-la. 0 valor de $\eta$, nesse caso, é denominado $\eta_{\infty}$, para um dado valor de $\beta$.

A Equação 07 é uma equação diferencial ordinária não linear. A condição de não linearidade da equação e a condição de aproximação na camada limite são os grandes desafios para obtenção da solução com razoável precisão. É uma equação com dois pontos como condição de contorno, o que leva a uma solução iterativa do tipo método do chute, método de Newton-Raphson ou método da bissecção. É importante ressaltar o interesse nas soluções positivas e nula da equação e, portanto, dentro do intervalo já definido acima para o parâmetro $\beta$. A situação especial, onde ocorre deslocamento, leva a uma raiz nula.

Em função da dificuldade em se resolver o problema de condição de contorno acima com razoável precisão, aplica-se o Método de Runge Kutta (TANNEHILL et al., 1997), com valor inicial de $f^{\prime \prime}(0)$ fornecido após aplicação do Método de Série de Potência.

A solução aproximada pelo método da série de potência, com aplicação do "Shooting Method" Tannehill et al. (1997), como procedimento de aproximação para o perfil de velocidade, é obtida admitindo-se que a função $f(\eta)$ satisfaça a seguinte expansão em série:

$$
f(\eta)=C_{2} \frac{\eta^{2}}{2 !}+C_{5} \frac{\eta^{5}}{5 !}+C_{6} \frac{\eta^{6}}{6 !} \ldots+C_{n} \frac{\eta^{n}}{n !}
$$

Com a seguinte regra de recorrência:

$$
\begin{aligned}
& C_{n+3}=-n !\left[\frac{1}{1 !(n-1 !)}\left(\beta \cdot C_{2} \cdot C_{n}\right)+\frac{1}{2 !(n-2 !)}\left(C_{2} \cdot C_{n}+\beta \cdot C_{3} \cdot C_{n-1}\right)+\frac{1}{3 !(n-3 !)}\left(C_{3} \cdot C_{n-1}\right)+\right. \\
& \left.\frac{1}{4 !(n-4 !)}\left(C_{5} \cdot C_{n-3}\right)\right]
\end{aligned}
$$

onde $n \geq 4$

$$
C_{3}=-\beta ; C_{5}=-2\left(\beta+\frac{1}{2}\right) C_{2}{ }^{2} \text { e } C_{6}=6 \beta\left(\beta+\frac{1}{2}\right) C_{2}
$$

o termo $C_{2}$ corresponde a $f^{\prime \prime}(0)$, ou seja:

$$
C_{2}=f^{\prime \prime}(0)
$$

Através do "Shooting Method" ou outro método de aproximação, como o "Método da Bissecção", podemos obter o valor de $C_{2}$, com aproximação desejada. 
A equação da energia, para determinação do campo de temperatura adimensional, é dada por:

$$
\frac{d}{d \eta}\left(\theta^{\prime}\right)+\operatorname{Pr} . f . \theta^{\prime}=0
$$

O perfil de temperatura deve satisfazer as seguintes condições de contorno, para temperatura especificada na superfície:

$$
\begin{array}{ll}
\eta=0, & \theta=0 \\
\eta \rightarrow \infty, & \theta \rightarrow 1.0
\end{array}
$$

onde Pr é o número de Prandtl, e

$$
\theta=\frac{T-T_{W}}{T_{\infty}-T_{W}}
$$

Assume-se que $T_{\infty}$, temperatura da corrente livre, não é afetada pela taxa de calor removida para a camada-limite. 0 valor de $T_{W}$ corresponde à temperatura na superfície.

A equação da energia, Equação 14, é linear e menos complexa que a equação do campo de velocidade. Entretanto, depende fortemente da solução do perfil de velocidade, uma vez que $f$ aparece explicitamente no segundo termo. Logo, quão maior a precisão na solução de $f$, melhor a solução em $\theta$.

Utilizou-se o método de Runge-Kutta para solução do campo de temperatura, mas observa-se que o valor limite para $\eta, \eta_{\infty}$ não é necessariamente o mesmo que o obtido para o campo de velocidade, para um dado $\beta$. Como alternativa, em termos de comparação, obteve-se uma segunda solução integrando diretamente a equação da energia. Logo, Kays and Crawford (1983) e Evans (1968):

$$
\begin{aligned}
& \theta=\theta_{0}^{\prime} \cdot \int_{0}^{\eta} \exp \left[-\operatorname{Pr} \cdot \int_{0}^{\eta} f \cdot d \eta\right] d \eta \\
& \theta^{\prime}=\theta_{0}^{\prime} \cdot \exp \left[-\operatorname{Pr} \cdot \int_{0}^{\eta} f \cdot d \eta\right]
\end{aligned}
$$

onde $\theta_{0}^{\prime}$ é o valor da derivada da temperatura na superfície da placa. Logo, como

$$
\theta \rightarrow 1, \quad \eta \rightarrow \infty \quad \theta_{0}^{\prime}=\frac{1}{\int_{0}^{\infty} \exp \left[-P r \cdot \int_{0}^{\eta} f \cdot d \eta\right] d \eta}
$$

Entretanto, a aplicação da Equação 18, acima, não possibilita uma precisão adequada para obtenção do gradiente de temperatura na superfície. Nesse sentido, opta-se por utilizar procedimento preconizado por Evans (1968), na determinação de $\theta_{0}^{\prime}$. 
Assim,

$\left(\frac{d \theta}{d \eta}\right)_{0}=\frac{3}{E}\left(\frac{\operatorname{Pr} \cdot f_{0}^{\prime \prime}}{3 !}\right)^{1 / 3}$

onde

$E=\Gamma\left(\frac{1}{3}\right)+\sum_{q=0}^{\infty} \frac{a_{q}}{\operatorname{Pr}^{\frac{q}{3}}}$

Гé a função gama, e:

$\Gamma\left(\frac{1}{3}\right)=2.6789385$

Expressões para $a_{q}$ contêm o parâmetro do gradiente de pressão $\beta$, a tensão viscosa adimensional na parede $f^{\prime \prime}(0)$, e fatores numéricos derivados da combinação das funções gama, porém não iremos apresentá-las aqui. O procedimento completo para a determinação encontra-se em Evans (1968). Ressalta-se, entretanto, que a Equação 19, para determinação do gradiente de temperatura na parede, apresenta resultados insatisfatórios, à medida que $\beta$ tende para o valor de separação ao escoamento $(\beta=-0.2)$. Evans (1968) descreve o procedimento alternativo para esse caso especial, mas ele também não será objeto de discussão nessa análise.

Para efeito de comparação, determinou-se grandezas equivalentes para escoamento turbulento em superfícies inclinadas lisas, através de um modelo aproximado, utilizando-se escoamento turbulento com potência 1/7, e resultados experimentais de Schultz-Grunow (1941), Pimenta et al. (1975), Schlicthing e Prandtl (1968), Kays and Crawford (1983). O procedimento teórico, nesse caso, corresponde ao preconizado por Kays e Crawford (1983), no qual as condições de similaridade são satisfeitas.

Para determinação aproximada do perfil de velocidade turbulento, associado à equação integral da quantidade de movimento, uma lei de potência do tipo 1/7 é deveras conveniente:

$$
u^{+}=8.75 y^{+1 / 7}
$$

A expressão acima representa o perfil de velocidade até $y^{+}=1500$ um pouco melhor do que a equação, muito utilizada em simulações algébricas, denominada "Lei Logarítmica na Parede".

$$
u^{+}=\frac{u}{\sqrt{\tau_{w} / \rho}} \quad y^{+}=y \frac{\sqrt{\tau_{w} / \rho}}{v}
$$

A equação integral da quantidade de movimento, em coordenadas similares, é dada por:

$$
f^{\prime \prime}(0)=\frac{1}{\delta_{4}}=\frac{1}{v U} \frac{d}{x}\left(U^{2} \delta_{2}\right)+\frac{\delta_{1}}{v} \frac{d U}{d x}
$$

ou

$$
\frac{\delta_{2}}{\delta_{4}}=\frac{1}{2} \frac{U}{v} \frac{d \delta_{2}{ }^{2}}{d x}+(2+H) \frac{\delta_{2}{ }^{2}}{v} \frac{d U}{d x}
$$


Onde

$\mathrm{H}=\frac{\delta_{1}}{\delta_{2}}$ é denominado fator de forma

Após manipulações algébricas, é possível demonstrar que:

$$
f^{\prime \prime}(0)=\beta \delta_{1}+(\beta+1) \delta_{2}
$$

Para camada limite similar, cada $\delta_{n}$ é uma constante e, portanto, o fator de forma é uma constante. É importante ressaltar que a Equação 25 é válida para regime laminar e turbulento. 0 fator de forma aumenta em um campo de pressão adverso, $\beta<0$. Para escoamento em camada limite turbulenta, $\mathrm{H}$ aumenta de, aproximadamente, 1,4 para gradiente de pressão nulo $\beta=0$, para, aproximadamente, 2,7 na separação (SIMPSON, 1989), $\beta \cong 0,2$. Para escoamento acelerado o valor de $\mathrm{H}$ aumenta novamente, em função do efeito de laminarização, e tende para 1.47 para escoamento bidimensional de estagnação, $\beta=1$ (SMITH, 1966). A distribuição de velocidade, $\mathrm{U}(\mathrm{x})$, deve ser conhecida antes da aplicação da equação integral da quantidade de movimento, Equação 24.

A espessura de deslocamento, $\delta_{1}$, tem o efeito de deslocar a função corrente do fluxo principal, não perturbado, com respeito ao valor que deveria ter para fluido ideal, não viscoso. A espessura de quantidade de movimento, $\delta_{2}$, é a medida pela qual a quantidade de movimento do fluido, na camada limite, está abaixo do que deveria ser para um fluido ideal. A espessura viscosa, $\delta_{4}$, inversa de $f^{\prime \prime}(0)$ , é a medida da resistência oferecida para a transferência da quantidade de movimento da corrente principal para a superfície.

Há duas regiões predominantes a serem analisadas em uma camada-limite turbulenta:

3. Uma região predominantemente viscosa próxima à superfície, onde imperam as tensões viscosas e a condução molecular;

4. Uma região completamente turbulenta, onde a quantidade de movimento e o calor são transportados em taxas geralmente muito superiores à da subcamada viscosa.

É na subcamada viscosa, entretanto, onde os eventos associados à turbulência ocorrem e tem maior importância do que a região completamente turbulenta. As forças viscosas, largamente responsáveis pelas características do escoamento laminar, tem o efeito de restaurar o escoamento laminar em escoamento turbulento e, de outra forma, as forças de inércia, associadas às variações locais do campo de velocidade, têm o efeito oposto. De fato, as forças de inércia tendem a amplificar os distúrbios locais. É de se esperar, portanto, que a estabilidade do escoamento laminar esteja associada a baixos números de Reynolds, razão entre as forças de inércia pelas forças viscosas. Apesar da instabilidade ser uma característica essencial na subcamada viscosa, a estrutura em camada limite turbulenta se auto ajusta, construindo uma estrutura relativamente estável e com características de estabilidade (existe regularidade!). Em escoamento turbulento, ao longo da superfície, a subcamada laminar vai ficando estreita e torna-se uma fração cada vez menor de toda a camada-limite. Em essência, a camada limite turbulenta possui a propriedade de difundir a quantidade de movimento e outras propriedades do escoamento, muito mais rapidamente do que o simples processo molecular. 
A Equação 22, junto com a equação integral da quantidade de movimento, Equação 24, pode ser utilizada para obter o coeficiente de fricção em camada-limite turbulenta. Note, entretanto, que o perfil de velocidade é válido para gradiente de pressão nulo, ou seja $\beta=0$. Para situações em que o gradiente de pressão é diferente de zero, deve-se efetuar algum tipo de correção. A expressão para o coeficiente de fricção, $\beta=0$, é dada por:

$$
\frac{C f}{2}=\frac{0.0594}{2 \operatorname{Re}_{x}^{1 / 5}}
$$

que pode ser comparada com a equação experimental obtida por Schultz-Grunow:

$$
\frac{C f}{2}=0.185\left(\log _{10}\left(R e_{x}\right)\right)^{-2.584}
$$

$\mathrm{Na}$ análise de camada-limite turbulenta é conveniente definir algum tipo de similaridade. Entretanto, a tarefa não é tão simples como em camada-limite laminar. Em escoamento turbulento, em uma região muito próxima da superfície, observa-se que $u^{+}=y^{+}$e, logicamente, o princípio de similaridade se aplica. Entretanto, fora dessa região e nesse sistema de coordenadas, o princípio geralmente não se aplica. Entretanto, existem algumas classes de escoamento que possuem algum tipo de similaridade, mesmo fora da subcamada laminar.

Camada-limite turbulenta que possui similaridade fora da subcamada laminar é denominada camada-limite em equilíbrio. Camada-limite em equilíbrio é a que satisfaz o seguinte perfil de velocidade:

$$
\begin{aligned}
& \frac{u-U}{\sqrt{\frac{\tau_{w}}{\rho}}}=F\left(\frac{y}{\delta_{3}}\right) \\
& \text { onde } \\
& \delta_{3}=-\int_{0}^{\infty} \frac{u-U}{\sqrt{\frac{\tau_{w}}{\rho}}} d y
\end{aligned}
$$

Para a camada-limite laminar foi demonstrado que a Equação 01 deve ser satisfeita para que soluções de similaridade existam. Em camada-limite turbulenta, esse mesmo tipo de perfil de velocidade para corrente livre deve ser satisfeito, para que ocorra camada-limite em equilíbrio, satisfazendo o princípio de similaridade (KAYS E CRAWFORD, 1983).

O efeito de um gradiente de pressão adverso é diminuir o coeficiente de fricção. A redução do coeficiente de friç̧ão, para camada-limite em equilíbrio, pode ser correlacionado com $\beta$ através de uma relação empírica (KAYS E CRAWFORD, 1983):

$$
\frac{C f / 2}{(C f / 2)_{\beta=0}}=\frac{1}{\left(1+\frac{\beta}{5}\right)}
$$

Um gradiente de pressão favorável, $\beta<0$, leva a um crescimento no valor do coeficiente de fricção. Entretanto, para $\beta$ muito baixo, próximo à região de separação, há uma forte aceleração do escoamento e ocorre uma camada-limite assintótica, e um efeito de laminarização do perfil de velocidade. 
Para camada-limite turbulenta, supondo camada-limite em equilíbrio, o número de Stanton é determinado, para gradiente de pressão nulo, através da expressão:

$$
S t_{x}=\frac{C f / 2}{\sqrt{C f / 2}(13.2 P r-10.16)+0.9}
$$

\section{RESULTADOS}

\subsection{Regime Laminar.}

Figura 3 - Perfil de velocidade adimensional para superfície inclinada.

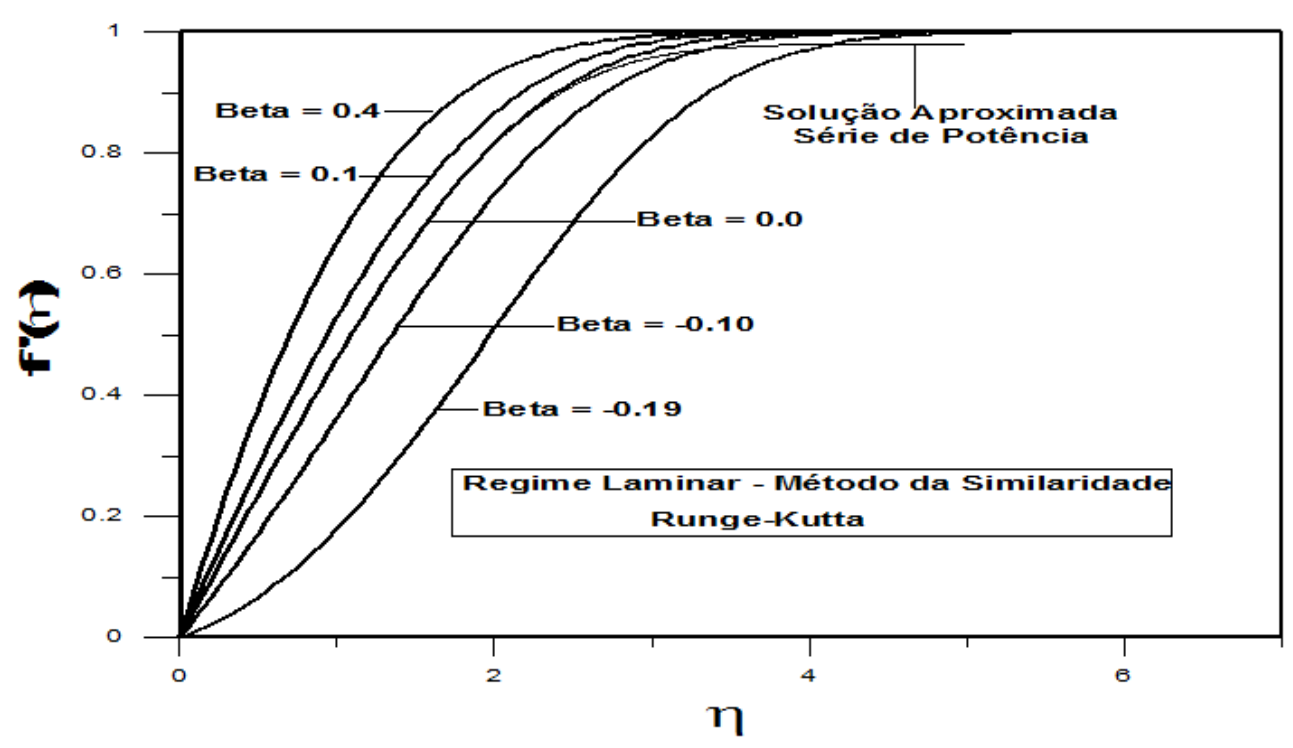

Fonte: autores, 2019

A Figura 03 , acima, apresenta os resultados obtidos para perfil de velocidade adimensional pelo método de Runge-Kutta. Temos, nesse caso, soluções para escoamento acelerado $(\beta>0)$ e escoamento retardado $(\beta<0)$. Como se pode observar, a solução pelo método de série de potência é obtida como primeira aproximação, antes de aplicarmos a solução pelo método de Runge-Kutta. É importante ressaltar, mais uma vez, que a precisão nesses resultados é de extrema importância para a determinação de parâmetros associados ao campo de velocidade, assim como para o campo de temperatura.

A Figura 04, abaixo, apresenta resultados para situações extremas, com $\beta=-0.2$ e $\beta=1.0$ . Tais condições representam, respectivamente, a condição de separação do escoamento e a condição de escoamento bidimensional de estagnação. Observe que, como esperado, para $\beta=-0.2$, a tensão viscosa é igual a zero na superfície. Para efeito de comparação, apresentamos os resultados para placa plana, $\beta=0$. 
Figura 04 - Soluções para perfil de velocidade e tensão viscosa adimensional para condições extremas: condição de separação $\beta=-0.2$ e condição de escoamento de estagnação bidimensional

$$
\beta=1.0 \text {. }
$$

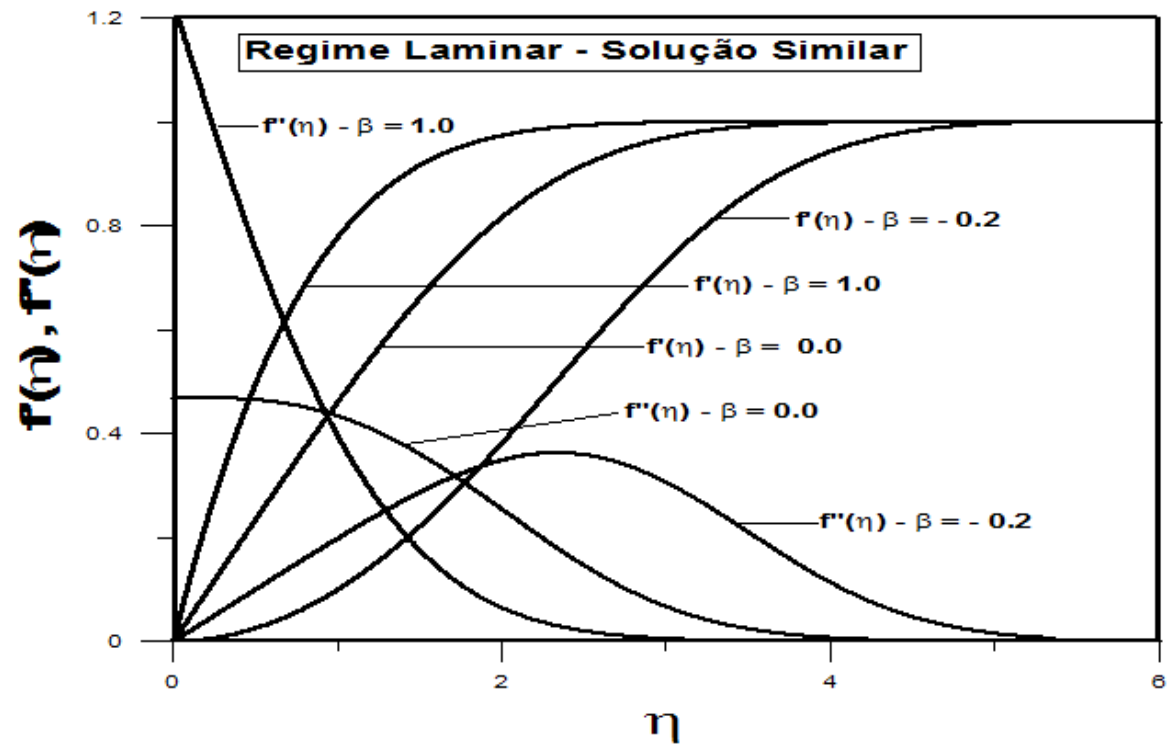

Fonte: autores, 2019.

Tabela 1 - Comparação entre parâmetros associados à camada-limite hidrodinâmica em Regime Laminar.

\begin{tabular}{ccccccccccc}
\hline \multicolumn{1}{c}{ Resultados } & \multicolumn{3}{c}{ Evans $^{*}$} & \multicolumn{3}{c}{ Stemmer } \\
\hline$\beta$ & $\delta_{1}$ & $\delta_{2}$ & $\mathbf{H}_{12}$ & $\mathbf{H}_{21}$ & $\delta_{1}$ & $\delta_{2}$ & $\mathbf{H}_{21}$ & $\delta_{1}$ & $\delta_{2}$ & $\mathbf{H}_{12}$ \\
-0.2 & 2.3587 & 0.5852 & 4.0306 & 0.2400 & $2.3588^{* *}$ & 0.5854 & 0.2482 & $2.358885^{* *}$ & 0.58544 & 4.0292 \\
-0.195 & 2.1167 & 0.5812 & 3.6419 & 0.2697 & 2.1170 & 0.5814 & 0.2746 & 2.11705 & 0.58136 & 3.64153 \\
-0.019 & 2.0064 & 0.5762 & 3.5701 & 0.2834 & 2.0068 & 0.5765 & 0.2873 & 2.00676 & 0.57652 & 3.48079 \\
-0.18 & 1.8714 & 0.5673 & 3.2988 & 0.3006 & 1.8716 & 0.5677 & 0.3033 & 1.87157 & 0.56771 & 3.29670 \\
-0.17 & 1.7785 & 0.5592 & 3.1804 & 0.3124 & 1.7789 & 0.5597 & 0.3146 & - & - & - \\
-0.16 & 1.7063 & 0.5520 & 3.0911 & 0.3218 & 1.7066 & 0.5522 & 0.3235 & - & - & - \\
-0.15 & 1.6468 & 0.5449 & 3.0222 & 0.3295 & 1.6470 & 0.5452 & 0.3310 & 1.64697 & 0.54518 & 3.02094 \\
-0.14 & 1.5956 & 0.5385 & 2.9630 & 0.3319 & 1.5959 & 0.5386 & 0.3375 & - & - & - \\
-0.12 & 1.5111 & 0.5258 & 2.8739 & 0.3472 & 1.5113 & 0.5263 & 0.3482 & 1.51134 & 0.52627 & 2.87178 \\
-0.10 & 1.4423 & 0.5147 & 2.8022 & 0.3562 & 1.4427 & 0.5150 & 0.3570 & 1.44307 & 0.51503 & 2.80190 \\
-0.05 & 1.3121 & 0.4901 & 2.6778 & 0.3732 & 1.3124 & 0.4905 & 0.3737 & - & - & - \\
0.0 & 1.2164 & 0.4695 & 2.5908 & 0.3856 & 1.2168 & 0.4696 & 0.3859 & 1.21678 & 0.4696 & 2.59110 \\
0.05 & 1.1416 & 0.4512 & 2.5301 & 0.3920 & 1.1417 & 0.4514 & 0.3954 & - & - & - \\
0.1 & 1.0831 & 0.4351 & 2.4893 & 0.4029 & 1.0832 & 0.4355 & 0.4031 & - & - & - \\
0.2 & 0.9839 & 0.4081 & 2.4087 & 0.4147 & 0.9842 & 0.4082 & 0.4148 & 0.98416 & 0.40823 & 2.41079 \\
0.3 & 0.9108 & 0.3856 & 2.3620 & 0.4234 & 0.9110 & 0.3857 & 0.4234 & 0.91099 & 0.38574 & 2.366167 \\
0.4 & 0.8525 & 0.3666 & 2.3254 & 0.4300 & 0.8527 & 0.3667 & 0.4301 & 0.85263 & 0.36669 & 2.32521 \\
0.5 & 0.8046 & 0.3503 & 2.2969 & 0.4354 & 0.8045 & 0.3503 & 0.4354 & - & - & - \\
0.6 & 0.7639 & 0.3359 & 2.2742 & 0.4397 & 0.7640 & 0.3359 & 0.4397 & 0.76397 & 0.33591 & 2.27435 \\
0.8 & 0.6986 & 0.3119 & 2.2398 & 0.4464 & 0.6987 & 0.3118 & 0.4463 & 0.69868 & 0.31185 & 2.24046 \\
1.0 & 0.6480 & 0.2924 & 2.2161 & 0.4514 & 0.6480 & 0.2923 & 0.4513 & 0.64790 & 0.29235 & 2.21618 \\
\hline & & & & & & & & & & -
\end{tabular}

Fonte: autores, 2019. 
A Tabela 01 apresenta comparações entre os resultados obtidos nessa análise e resultados apresentados por Evans, para os parâmetros associados ao escoamento adimensional pelo método da similaridade, onde:

$$
\begin{aligned}
& \delta_{1}=\int_{0}^{\infty}\left(1-f^{\prime}\right) d \eta \\
& \delta_{2}=\int_{0}^{\infty} f^{\prime}\left(1-f^{\prime}\right) d \eta \\
& H_{21}=\frac{1}{H_{12}}=\frac{\delta_{2}}{\delta_{1}}
\end{aligned}
$$

$\delta_{1}$ é a espessura de deslocamento em variáveis similares e $\delta_{2}$ é a espessura de quantidade de movimento. Os resultados obtidos para $\beta$ próximo da unidade estão em excelência concordância, e os resultados próximos do descolamento da camada-limite estão razoáveis, demonstrando que os perfis estão definidos com ótima precisão.

Figura 5 - Perfil de temperatura e gradiente de temperatura para camada limite térmica em regime laminar e número de Prandtl igual a unidade.

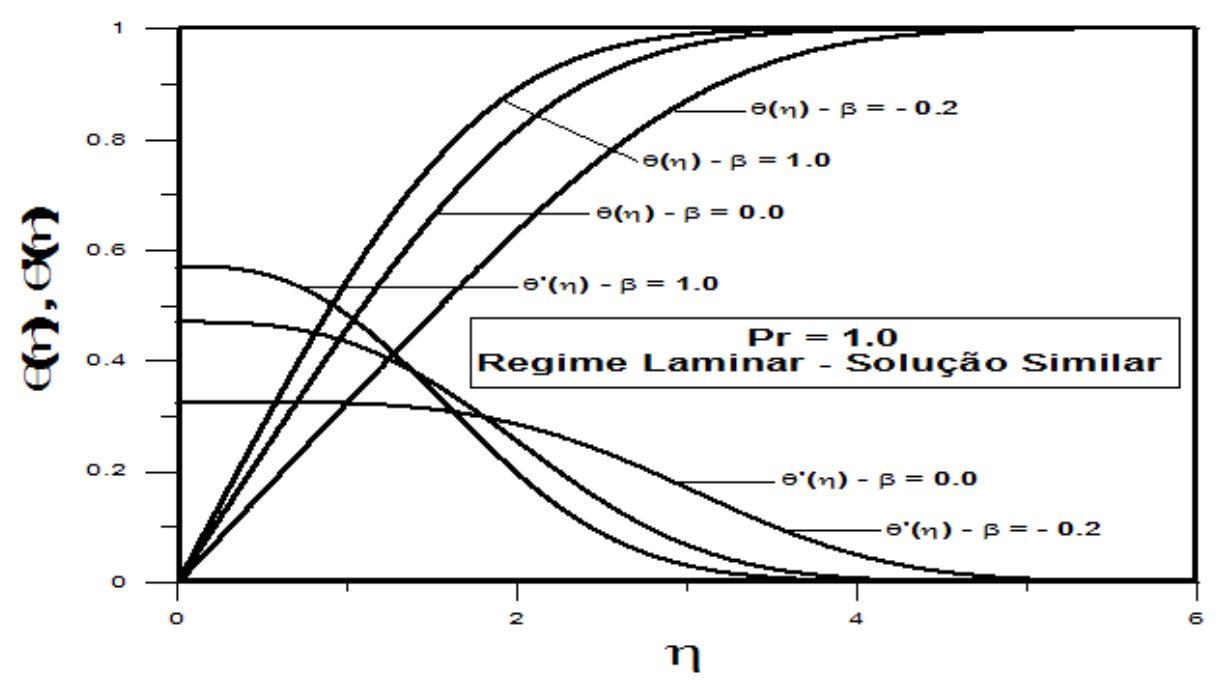

Fonte: autores, 2019.

Na Figura 05, temos os resultados para perfil de temperatura adimensional e gradiente de temperatura para $\operatorname{Pr}=1.0$, em regime laminar. As soluções estão representadas para condições limítrofes, $\beta=-0.2$ e $\beta=1.0$, com placa plana como contraponto. Os resultados mais importantes, nesse caso, são os valores do gradiente de temperatura na superfície, uma vez que os parâmetros associados ao campo de temperatura encontram-se fortemente associados a ele. Como se pode observar através da Equação 19, o gradiente de temperatura na superfície está vinculado à tensão viscosa na parede e depende, portanto, da solução do campo de velocidade. A Figura 06 mostra a variação do gradiente de temperatura ao longo da camada-limite, para número de Prandtl igual 0,7 e tendo o gradiente de pressão como referência. 
Figura 6 - Gradiente de temperatura na camada-limite térmica em função do gradiente de pressão e $\operatorname{Pr}=0.7$, em regime laminar.

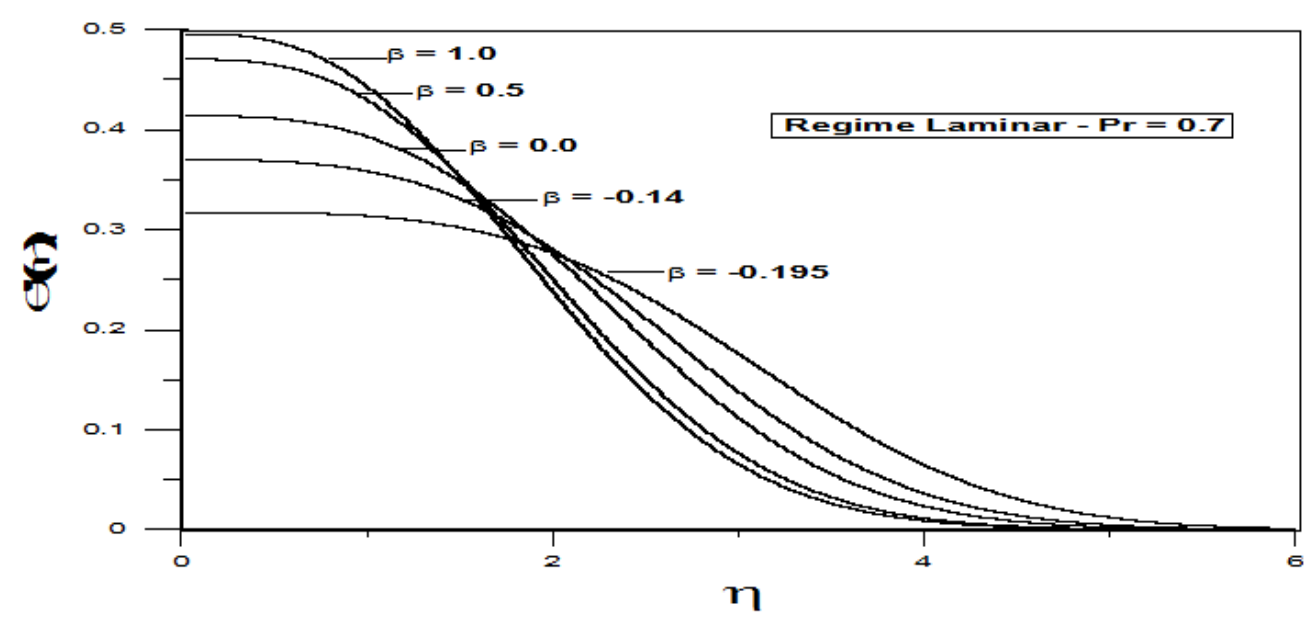

Fonte: autores, 2019.

Figura 7 - Número de Stanton em função do número de Reynolds para $\beta=1.0$, com variação do número de Prandtl entre 0.7 e 10.

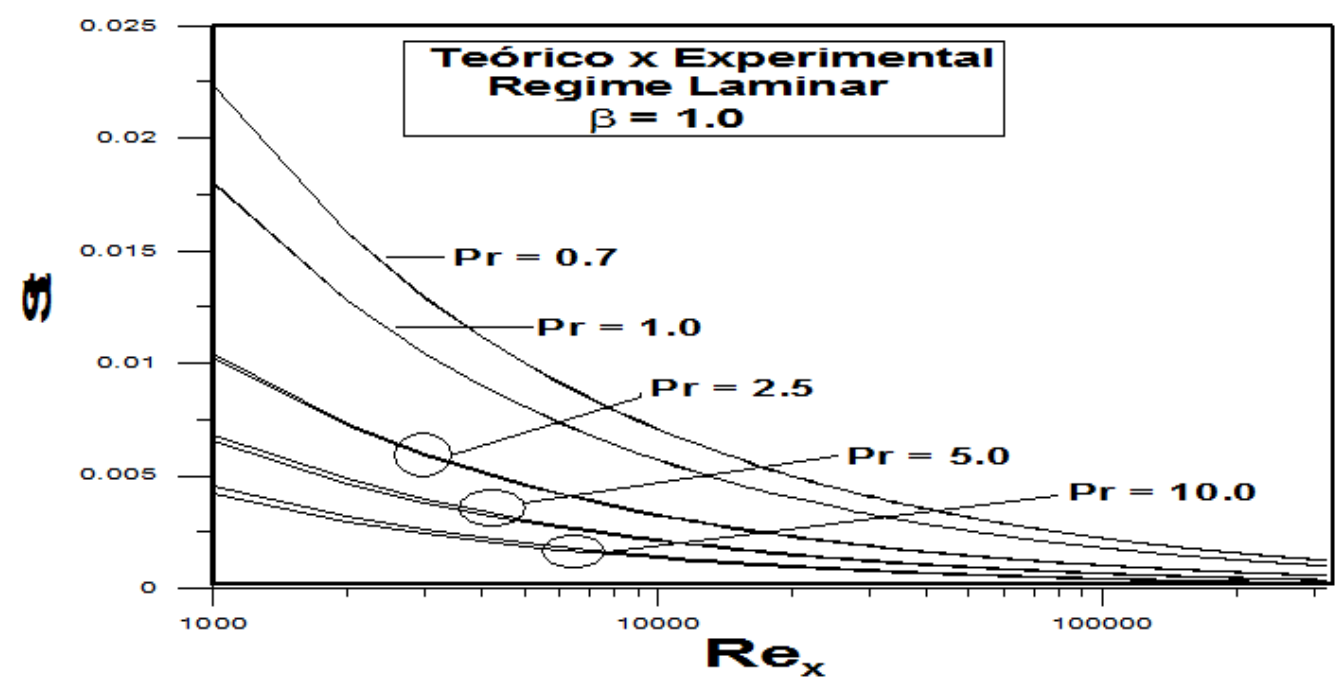

Fonte: autores, 2019.

Na Figura 07, apresentamos comparação teórico-experimental para número de Stanton, em regime laminar e escoamento de estagnação bidimensional, e número de Prandtl próximo da unidade. A equação para o cálculo do número de Nusselt experimental é dada por:

$$
N u=0.57 \operatorname{Re}_{x}^{1 / 2} \operatorname{Pr}^{0.4}
$$

Observe que, para valores próximos da unidade, $\beta=0.7$ e $\beta=1.0$, os resultados teóricos e experimentais coincidem e sofrem ligeira variação para valores superiores à unidade. 


\subsection{Regime turbulento}

\subsubsection{Superfície lisa}

Figura 8 - Coeficiente de fricção para regime turbulento, em função do parâmetro de gradiente de pressão.

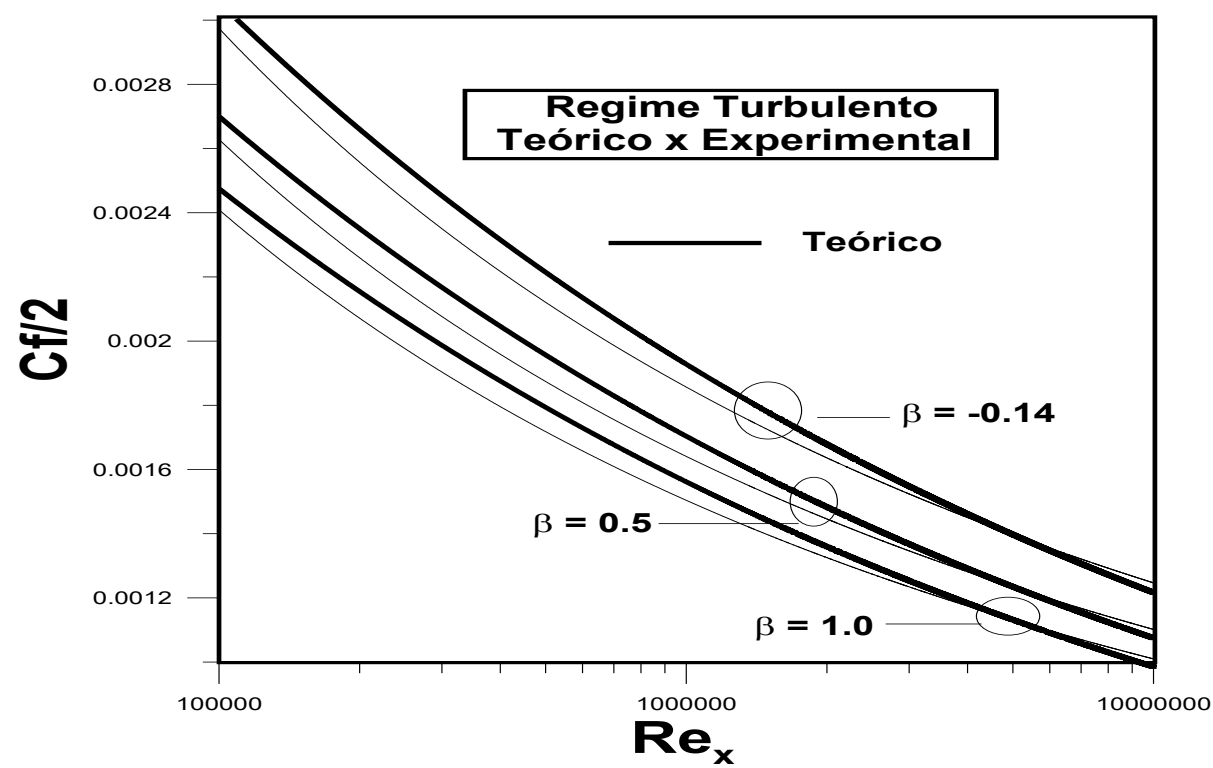

Fonte: autores, 2019

$\mathrm{Na}$ Figura 08, apresentamos resultados para fator de friç̧ão em regime turbulento. Os resultados em destaque demonstram as comparações com resultados experimentais obtidos por Schultz-Grunow (1941), aplicando-se o fator de correção $(1+\beta / 5)$. O coeficiente de fricção, nesse caso, é obtido por extensão dos desenvolvimentos apresentados por Kays e Crawford(1983), através do método integral da quantidade de movimento e de corretor para superfície inclinada:

$$
\frac{C f}{2}=\frac{0.0594}{2 R e_{x}^{1 / 5} *\left(1+\frac{\beta}{5}\right)}
$$

A expressão experimental é determinada pela expressão de Schultz-Grunow e correção mencionada:

$$
\left(\frac{C f}{2}\right)_{E x p}=\left[0.185\left(\log _{10}\left(R e_{x}\right)\right)^{-2.584}\right] /\left(1+\frac{\beta}{5}\right)
$$

Na Figura 09, admitimos uma variação para $H_{12}$ turbulento equivalente à variação para regime laminar, uma vez que ocorre o efeito de laminarização para escoamento altamente acelerado e completamente turbulento. De fato, os resultados apresentados mostram que, para escoamento acelerado, os valores se aproximam do resultado obtido para escoamento laminar (em destaque). Esse efeito é denominado de laminarização da camada limite (KAYS et al., 1969) e demonstram que o efeito da aceleração tende a causar uma "retransição" da camada-limite turbulenta para uma pura camada-limite laminar. 
Figura 9 - Comparação para Fator de Forma entre regime laminar e regime turbulento

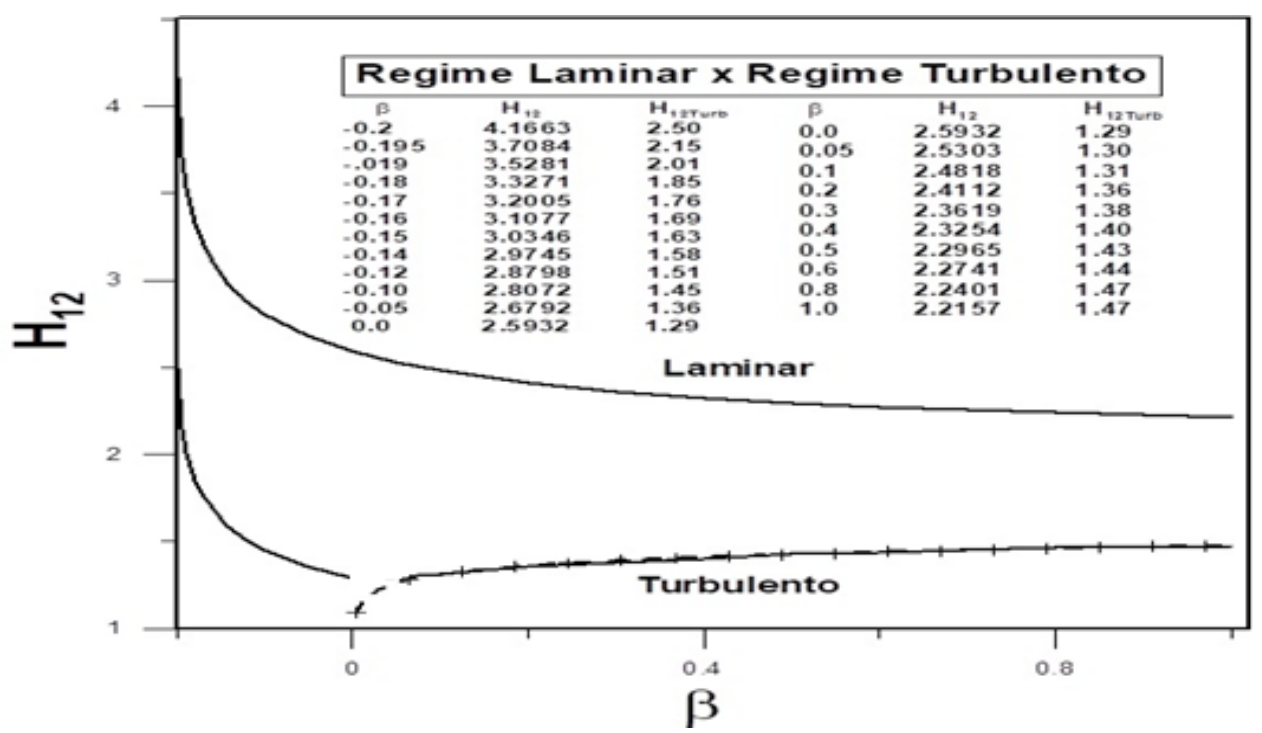

Fonte: autores, 2019.

A expressão obtida para cálculo do fator de forma para regime turbulento é dada por:

$$
\begin{aligned}
& H_{12 \text { Turb }}=\frac{\left[(2.7-1.29) *\left(H_{12}-2.5932274\right)\right]}{[(4.166269-2.5932274)]}+1.29, \quad-0.2 \leq \beta \leq 0.0 \\
& H_{12 \text { Turb }}=\frac{\left[(1.47-1.29) *\left(H_{12}-2.21573244\right)\right]}{[(2.21573244-2.5932274)]}+1.47, \quad 0.0<\beta \leq 1.0
\end{aligned}
$$

Entretanto, devido à alteração abrupta nos parâmetros do escoamento, uma vez que ocorre um processo de aceleração e laminarização, como já discutido, uma continuidade em zero é pouco provável e uma solução alternativa, para valores de $\beta$ positivo, é assumir uma variação do tipo:

$$
y=B \ln (\beta)+A, \text { onde } B=0.069487 \text { e } A=1.47
$$

A Equação (42) resgata os valores de $\mathrm{H}_{12 \mathrm{Turb}}$ da tabela apresentada na Figura 09, para $\beta \geq 0.1$, e os resultados encontram-se em destaque no gráfico.

Para regime turbulento, superfície lisa, o número de Stanton é determinado por:

$$
S t_{T u r b}=\frac{N u_{\text {Turb }}}{\left(\operatorname{Pr} * \operatorname{Re} e_{x}\right)\left(1.0+\frac{\beta}{5}\right)}
$$

onde,

$$
N u_{\text {Turb }}=0.0296 * \operatorname{Re}_{x}^{0.8} * \operatorname{Pr}\left(\frac{1}{3}\right)
$$


Figura 10 - Número de Stanton em função do número de Reynolds, com variação do coeficiente de pressão em regime turbulento.

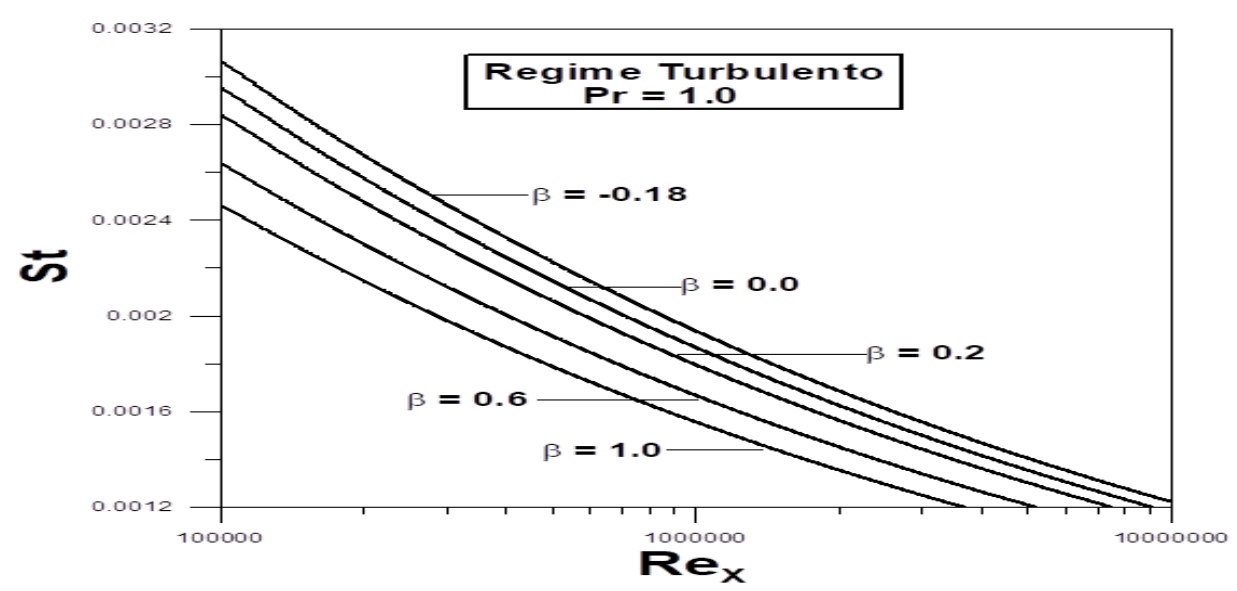

Fonte: autores, 2019

A Figura 11 apresenta resultados para número de Stanton em função do número de Reynolds, superfície lisa, com número de Prandtl unitário.

Figura 11 - Comparação teórico-experimental para número de Stanton em regime turbulento, com número de Prandtl unitário e $5.10^{5}<R e_{x}<5.10^{6}$.

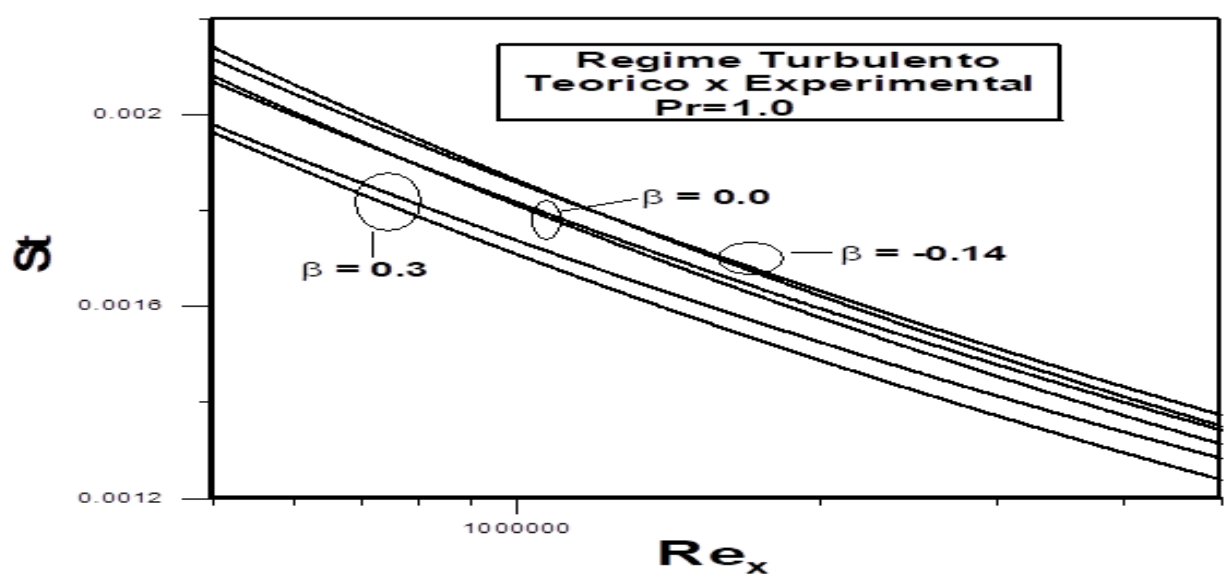

Fonte: autores, 2019

Em qualquer situação, regime laminar ou turbulento, para temperatura da superfície constante e velocidade da corrente livre constante, o número de Stanton pode ser expresso na forma:

$$
\begin{aligned}
& \text { St } \\
& =C e_{x}{ }^{-n}
\end{aligned}
$$

Em regime turbulento, gradiente de pressão nulo, Kays e Crawford apresentam a seguinte equação, que se ajusta de forma excelente com resultados experimentais para $0.5<\operatorname{Pr}<1.0$ e $5.10^{5}<\operatorname{Re}_{x}<5.10^{6}: C=0.0287 \operatorname{Pr}^{-04}$ e $n=0.20$. 
Introduzindo a correção proposta para superfícies inclinadas, temos:

$$
\begin{aligned}
& S t_{\text {TurbExp }} \\
& =\frac{0.0287 \mathrm{Pr}^{-0.4} \mathrm{Re}_{x}{ }^{-0.20}}{1+\frac{\beta}{5}}
\end{aligned}
$$

A Figura 11 apresenta resultados para número de Stanton, dentro da faixa de validade da expressão que se correlaciona de forma excelente com resultados experimentais para gradiente de pressão nulo. A comparação teórico-experimental demonstra que a expressão corrigida, Equação 46, se correlaciona de forma satisfatória com o modelo generalizado desenvolvido neste trabalho, Equação 43. A depressão ou decaimento do número de Stanton para escoamento acelerado é resultado de uma combinação do aumento na espessura da região viscosa, e o crescimento da camada-limite térmica além da camada-limite de quantidade de movimento (KAYS et al., 1969).

\section{CONCLUSÃO}

A análise efetuada, para escoamento e transferência de calor em regime laminar e regime turbulento, em superfície inclinada lisa, inclui aspectos teóricos e correlações empíricas experimentais. Há uma extensa revisão de procedimentos associados ao método da similaridade. Os aspectos relacionados com regime laminar estão associados, principalmente com os trabalhos de Harry L. Evans (1968) e os aspectos associados ao regime turbulento, superfície lisa, estão relacionados aos trabalhos de W. M. Kays e associados $(1983 ; 1975)$.

Em regime turbulento, através da análise integral da quantidade de movimento, as equações foram obtidas primeiramente para gradiente de pressão nulo e estendidas através de um fator de correção apresentado por Kays e Crawford (1983) para uma ampla faixa de gradiente de pressão.

Os resultados, quando comparados com equações empíricas e experimentais, se mostram qualitativamente consistentes e muito próximos dos apresentados na literatura. 


\section{REFERÊNCIAS}

ABBASI M. et al. "Analytic Solution of Hydrodynamic and Thermal Boundary Layer Over a Flat Plate in a Uniform Stream of a Fluid with Convective Surface Boundary Condition". Indian J.Sci.Res.1(2): 241 247, 2014.

ALI N, NAZEER M, TARIQ J, MUDASSAR R. "Finite element analysis of bi-viscosity fluid enclosed in a triangular cavity under thermal and magnetic effects". The Eur. Phys. J. 134:2, 2019.

BHATTACHARYYA, K. ET ALL "Exact Solution for Thermal Boundary Layer in Casson Fluid Flow over a Permeable Shrinking Sheet with Variable Wall Temperature and Thermal Radiation". Alexandria Engineering Journal (2016) 55, 1703-1712.

BLASIUS, H. "Grenzschichten in Flussigkeitein mit Kleiner Reibung." Z. Math, und Phys. 56, 1 (1908).

BOGNAR, G.; HRICZÓ. "Similarity Solutions a Thermal Boundary Layer Models of a non-Newtonian Fluid with a Convective Surface Boundary Condition". Acta Polytechnica Hungarica, Vol 8, No. 6, 2011.

CASTILHO, L. "Similarity Analyses of Turbulent Boundary Layer". A Dissertation Submitted in a Partial Requirement for the Degree of Doctor of Philosophy, State University of Buffalo, 1997.

EVANS, HARRY L. "Laminar Boundary-Layer Theory". ADDISON-WESLEY PUBLISHING COMPANY, 1968.

FALKNER, V. M. AND S. W. SKAN. "Some approximate solutions of the boundary layer equations." British Aero. Res. Council, Reports and Memoranda. 1314 (1930).

KHAN NA, KHAN S, ARA A. "Flow of micro polar fluid over an off centered rotating disk with modified Darcy's law". Propulsion Power Res. 6(4):285-295, 2017.

KAYS, W. M.; CRAWFORD, M. E. "Convective Heat and Mass Transfer". Tata McGraw-Hill Publising Co. Ltd, New Delhi, 1983.

MARTIN, M. J. et al. "Momentum and Heat Transfer in a Laminar Boundary Layer with Slip Flow". Journal of Thermophysics and Heat Transfer, Vol. 20, No. 4, October-December, 2006.

MYERS, T. G. "An Approximate Solution Method for Boundary Layer Flow of A Power Law Fluid Over a Flate Plate". International Journal of Het and Mass Transfer, 53 (2010) 2337-2346.

NAZEER M, ALI N, TARIQ J. "Numerical simulation of MHD flow of micropolar fluid inside a porous inclined cavity with uniform and non-uniform heated bottom-wall". Can. J. Phys. 96(6):576-593, 2017.

NOGUEIRA, ÉLCIO; SOARES, MARCUS VINICIUS FERREIRA. Camada-limite em placa plana: revisitando o Método da Similaridade. Cadernos UniFOA, Volta Redonda, n. 37, p. 15-31, ago. 2018.

ODERINU, R. A. "Shooting Method via Taylor Series for Solving Two Point Boundary Value Problem on an Infinite Interval". Gen. Math. Notes, Vol. 24, No. 1, September, pp. 74-83, 2014.

PIRES, L. B. MARINHO. "Estudo da Camada Limite Interna Desenvolvida em Falésias com Aplicação para o Centro de Lançamento de Alcântara". Tese de Doutorado do Curso de Pós-Graduação em Meteorologia, Inpe, São José dos Campos, 2009. 
RAHMAN, M. M. "Locally Similar Solutions for a Hydromagnetic and Thermal Slip Flow Boundary Layers over a Flate Plate with Variable Fluid Properties and Convective Surface Boundary Condition". Meccanica (2011) 46:1127-1143. DOI 10.1007/s11012-010-9372-2.

SCHULTZ-GRUNOW, F.: NA TM-986, Washington, 1941.

SCHLICHTING, H. McGraw-Hill Book Company. New York, 1968.

SHAMES, I. H. "Mecânica dos Fluidos: Análise de Escoamentos". Editora Edgard Blucher Ltda, Vol. 2, 1973. Tradução: Mauro O. C. Amorelli

SILVA, A. F. G.; FISCH, G. "Avaliação do Modelo WRF para a Previsão do Perfil do Vento no Centro de Lançamento de Alcântara". Revista Brasileira de Meteorologia, V. 29, n.2, 259-270, 2014.

SPALDING, D. B.; PUN, W. M. "A Review of Methods for Predicting Heat-Transfer Coefficients for Laminar Uniform-Property Boundary Layers Flows". Int. J. Heat Mass Transfer, Vol. 5, pp. 239-249, Pergamon Press 1962.

STEMMER, C. "Boundary Layer Theory: Falkner-Scan Solution (Flat-Plate Boundary Layer with Pressure Gradients)". Lehrstuhf für Aerodynamik, Tecnische Universität Münchem, Boundary Layer Theory, GIST 2010. 